\title{
BROKEN SUPERSYMMETRY FOR THE ELECTRON IN THE MAGNETIC FIELD OF STRAIGHT CURRENT
}

\author{
V. M. Tkachuk, S. I. Vakarchuk \\ The Ivan Franko National University of Lviv, \\ Chair of Theoretical Physics \\ 12 Drahomanov Str., Lviv, UA-79005, Ukraine
}

(Received October 28, 1999)

\begin{abstract}
The Pauli Hamiltonian for the electron moving in the magnetic field of straight current possesses $N=3$ supersymmetry (SUSY). We study the equation for the zero energy ground state of the electron moving in this field. It is shown that this equation does not have square integrable solutions and thus the SUSY is broken.
\end{abstract}

Key words: broken supersymmetry, quantum mechanics, magnetic field.

PACS numbers: 11.30.Pb, 03.65.-w

\section{INTRODUCTION}

Originally supersymmetry (SUSY) has been introduced into relativistic quantum field theory in order to unify bosons and fermions. Nowadays the notion of SUSY is successfully applied to different areas of theoretical physics such as atomic, nuclear, solid state and statistical physics. H. Nicolai was the first to show that SUSY could be a useful tool in nonrelativistic quantum mechanics [1]. Independently E. Witten introduced one dimensional SUSY quantum mechanics as a laboratory for investigating the SUSY breaking [2] which is one of the fundamental issues in the SUSY quantum field theory.

At the present time SUSY quantum mechanics is interesting on its own merit and has been studied from different points of view [3-5]. One of the quantum-mechanical problems where SUSY is the physical symmetry is the motion of the electron in the magnetic field. It was shown that $N=2$ SUSY is realized in the case of an arbitrary two-dimensional magnetic field $B_{x}=B_{y}=0$, $B_{z}=B_{z}(x, y)$ and the three-dimensional one which possesses the following symmetry with respect to inversion of coordinates: $\mathbf{B}(-\mathbf{r})= \pm \mathbf{B}(\mathbf{r})$ [4-7]. The field of the magnetic monopole is one of the examples where SUSY is realized in the three-dimensional case [6]. It was shown also that the electron motion on the surface orthogonal to the magnetic field possesses $N=2$ SUSY [8]. In our recent papers $[9,10]$ and the papers by Nikitin $[11,12]$ new three-dimensional magnetic fields in which the motion of the electron is supersymmetric were found. Another new aspect is that in the considered magnetic fields the SUSY with two, three and four supercharges is realized.

In $[10]$ we showed that the motion of the electron in the magnetic field of straight current possesses $N=3$ SUSY. For the electron moving in such (one of the simplest possible) magnetic configuration it is impossible to obtain the exact solution of the corresponding eigenvalue problem. In contrast to the charged particle the eigenvalue problem for the neutral spin $\frac{1}{2}$ particle moving in the magnetic field of the current-carrying wire can be solved exactly in different ways: by using supersymmetry in coordinate space [13], by differential equation techniques [14], and by using supersymmetry in momentum space [15]. Surprisingly, the energy spectrum in this case obeys a hydrogen Rydberg formula. The energy spectrum and eigenstates of the charged particle with the spin of $\frac{1}{2}$ in the magnetic field of the current-carrying wire was determined numerically in [16].

In the present paper we study the problem of the existence of exact or broken SUSY for the electron moving in the magnetic field of straight current. In order to answer this question it is necessary to investigate the equation for the ground state with the zero-energy level in the considered magnetic fields. If zero modes exist, then we have exact SUSY. If zero modes do not exist, the SUSY is broken. It is an interesting problem to ask how many solutions of the equation for zero modes exist. For twodimensional magnetic fields this problem was solved by Y. Aharonov and A. Kasher [19]. It was shown that the number of states with zero energy is equal to $\left[\Phi / \Phi_{0}\right]$ and $\Phi / \Phi_{0}-1$ if $\Phi / \Phi_{0}$ is integer, where $\Phi$ is the magnetic flux over the $X Y$ plane, $\Phi_{0}=2 \pi \hbar c /|e|$ is the flux quantum. As we know in the three-dimensional case no completely satisfactory answer to such a question exists. Some examples of the zero energy bound state in a threedimensional magnetic field have been obtained in [20] and further results have been provided recently in [21]. In these articles no degeneracy of zero modes has been observed. Recently in paper [22] it was proved that the phenomenon of degeneracy of zero modes does indeed occur in a thee-dimensional case. It should be emphasised that the problem of the existence of zero modes has some deep physical implications. In [23] it was proven that one-electron atom with sufficiently high nuclear charge in an external magnetic field is unstable if zero modes of the Pauli operator exist. Note that paper [20] was incited by paper [23].

In the present paper it will be shown that for the electron moving in the magnetic field of straight current the zero modes do not exist and thus SUSY is broken. 


\section{SUSY OF THE ELECTRON IN THE MAGNETIC FIELD OF STRAIGHT CURRENTS WITH AXIAL SYMMETRY}

The Pauli Hamiltonian for the electron moving in the magnetic field reads

$$
H=\frac{1}{2 m}\left(\mathbf{p}-\frac{e}{c} \mathbf{A}\right)^{2}-g \frac{e \hbar}{4 m c} \boldsymbol{\sigma} \mathbf{B},
$$

where $\sigma_{\alpha}$ are the Pauli matrices, $\mathbf{A}$ is the vector potential, $\mathbf{B}=\operatorname{rot} \mathbf{A}$ is the magnetic field. The Hamiltonian (1) can be rewritten in the following form

$$
H=Q_{0}^{2}-(g-2) \frac{e \hbar}{4 m c} \boldsymbol{\sigma} \mathbf{B},
$$

where

$$
Q_{0}=\frac{1}{\sqrt{2 m}} \boldsymbol{\sigma}\left(\mathbf{p}-\frac{e}{c} \mathbf{A}\right)
$$

Note that for the electron the gyromagnetic ratio $g$ only slightly differs from 2 , namely, $g=2.0023$. It is worthwhile stressing that taking into account the anomalous magnetic moment of the electron $(g>2)$ leads to the so-called anomalous electron trapping by the magnetic fields $[17,18]$. These problems are irrelevant for our paper. We put $g=2$. In this case the Pauli Hamiltonian possesses SUSY and $Q_{0}$ is called the supercharge.

In the present paper we consider the motion of the electron in the magnetic field of straight currents. Let us assume that the current is parallel to the $z$ axis with radial symmetry of the distribution of current density. The vector potential in this case reads

$$
A_{x}=A_{y}=0, \quad A_{z}=A(\rho),
$$

where $\rho=\sqrt{x^{2}+y^{2}}$.

Recently we showed that in this case the Pauli Hamiltonian possesses $N=3$ SUSY [10], namely, in addition to $Q_{0}$ we have two more supercharges

$$
Q_{1}=i \sigma_{x} I_{x} Q_{0}, \quad Q_{2}=i \sigma_{y} I_{y} Q_{0},
$$

where $I_{x}$ and $I_{y}$ are the inversion operators of the $x$ and $y$ axes respectively. We can easily check that the supercharges fulfil the following SUSY algebra

$$
\left\{Q_{\alpha}, Q_{\beta}\right\}=2 \delta_{\alpha, \beta}, \quad \alpha, \beta=0,1,2,
$$

$$
\left[Q_{\alpha}, H\right]=0 \text {. }
$$

As a result of the axial symmetry the $z$ component of the total angular momentum $J_{z}=L_{z}+S_{z}$ is the integral of motion and commutes with the Hamiltonian, i.e. $\left[J_{z}, H\right]=0$. In addition, $J_{z}$ satisfies the following permutation relations

$$
\begin{aligned}
& {\left[J_{z}, Q_{0}\right]=0,} \\
& \left\{J_{z}, Q_{1}\right\}=\left\{J_{z}, Q_{2}\right\}=0 .
\end{aligned}
$$

\section{BROKEN SUSY}

In order to answer the question about the existence of the exact or broken SUSY we shall investigate the equation for the zero energy ground state. Due to the axial symmetry it is convenient to rewrite the Hamiltonian in the polar coordinates

$$
\begin{array}{r}
H=-\frac{\hbar^{2}}{2 m} \frac{1}{\rho} \frac{\partial}{\partial \rho} \rho \frac{\partial}{\partial \rho}+\frac{1}{2 m} \frac{1}{\rho^{2}}\left(-i \hbar \frac{\partial}{\partial \phi}\right)^{2} \\
+\frac{1}{2 m}\left(-i \hbar \frac{\partial}{\partial z}-\frac{e}{c} A(\rho)\right)^{2}-g \frac{e \hbar}{4 m c} \boldsymbol{\sigma} \mathbf{B} .
\end{array}
$$

The coupling of the spin with the magnetic field depends on $\phi$

$$
\boldsymbol{\sigma} \mathbf{B}=A^{\prime}(\rho)\left(\sigma_{x} \sin \phi-\sigma_{y} \cos \phi\right)
$$

where $A^{\prime}(\rho)=\partial A(\rho) / \partial \rho$. This dependence can be removed using the unitary transformation

$$
\tilde{\psi}=e^{i \phi \sigma_{z} / 2} \psi
$$

where the new wave function satisfies the following condition

$$
\tilde{\psi}(\phi+2 \pi)=-\tilde{\psi}(\phi)
$$

As a result of the unitary transformation Eq. (10) becomes

$$
e^{i \phi \sigma_{z} / 2} \boldsymbol{\sigma} \mathbf{B} e^{-i \phi \sigma_{z} / 2}=-A^{\prime}(\rho) \sigma_{y} .
$$

For the Pauli Hamiltonian after the unitary transformation we obtain

$\tilde{H}=e^{i \phi \sigma_{z} / 2} H e^{-i \phi \sigma_{z} / 2}=-\frac{\hbar^{2}}{2 m} \frac{1}{\rho} \frac{\partial}{\partial \rho} \rho \frac{\partial}{\partial \rho}+\frac{1}{2 m} \frac{1}{\rho^{2}}\left(-i \hbar \frac{\partial}{\partial \phi}-S_{z}\right)^{2}+\frac{1}{2 m}\left(-i \hbar \frac{\partial}{\partial z}-\frac{e}{c} A(\rho)\right)^{2}+g \frac{e \hbar}{4 m c} A^{\prime}(\rho) \sigma_{y}$. 
Note that after the unitary transformation the operator $-i \hbar \frac{\partial}{\partial \phi}=\tilde{J}_{z}$ represents a new operator of the $z$ component of the total angular momentum. We can separate variables and represent the wave function as follows

$$
\tilde{\psi}=e^{i j_{z} \phi} e^{i k z} \frac{1}{\sqrt{\rho}} R(\rho)
$$

where $k$ is the wave vector of the electron motion along the $z$ axis, $j_{z}= \pm \frac{1}{2}, \pm \frac{3}{2}, \ldots$ is the eigenvalue of the $z$ component of the total angular momentum in the units of $\hbar$. The radial part $R(\rho)$ of the eigenfunction satisfies the conditions $R(0)=R(\infty)=0$. The equation for $R(\rho)$ reads

$$
H_{\rho} R(\rho)=\epsilon R(\rho)
$$

where the radial part of the Pauli Hamiltonian has the form

$$
\begin{aligned}
H_{\rho} & =-\frac{\partial^{2}}{\partial \rho^{2}}+\frac{1}{\rho^{2}}\left[\left(j_{z}-\frac{\sigma_{z}}{2}\right)^{2}-\frac{1}{4}\right] \\
& +(k-\gamma A(\rho))^{2}+\gamma A^{\prime}(\rho) \sigma_{y} .
\end{aligned}
$$

We introduced the notation $\gamma=e / \hbar c, \epsilon=2 m E / \hbar^{2}$.

The Hamiltonian $H_{\rho}$ can be written in the form

$$
H_{\rho}=Q^{2},
$$

where

$$
\begin{aligned}
Q & =-i \sigma_{x} \frac{\partial}{\partial \rho}+f \sigma_{z}+\frac{j_{z}}{\rho} \sigma_{y}, \\
f & =k-\gamma A(\rho) .
\end{aligned}
$$

At the beginning of the paper we chose the direction of the current along the $z$ axis. Sometimes it is convenient to consider the case when the current is directed along the other axis. Thus, when the current is directed along the $x$ axis the operator $Q$ is as follows

$$
Q=-i \sigma_{y} \frac{\partial}{\partial \rho}+f \sigma_{x}+\frac{j_{z}}{\rho} \sigma_{z}
$$

and it can be obtained from (20) using cyclic substitution of the Pauli matrices. Using once more the cyclic substitution we obtain $Q$ for the case when the current is directed along the $y$ axis

$$
Q=-i \sigma_{z} \frac{\partial}{\partial \rho}+f \sigma_{y}+\frac{j_{z}}{\rho} \sigma_{x}
$$

Now let us consider the equation for the zero energy ground state $\epsilon=0$ which due to (19) reads

$$
Q R=0
$$

It is convenient to consider $Q$ given by (21). Explicitly Eq. (23) is a set of two equations for the components $R_{1}$ and $R_{2}$ of the radial part of the wave function

$$
\begin{gathered}
\frac{j_{z}}{\rho} R_{1}+\left(-\frac{\partial}{\partial \rho}+f\right) R_{2}=0 \\
\left(\frac{\partial}{\partial \rho}+f\right) R_{1}-\frac{j_{z}}{\rho} R_{2}=0
\end{gathered}
$$

This set of the first order differential equations can be transformed to the second order differential equation for one of the components of the wave function. For $R_{1}$ and $R_{2}$ we obtain respectively

$$
\begin{gathered}
\left(-\frac{\partial^{2}}{\partial \rho^{2}}+f^{2}-f^{\prime}\right) R_{1}-\frac{1}{\rho}\left(\frac{\partial}{\partial \rho}+f\right) R_{1}+\frac{j_{z}^{2}}{\rho^{2}} R_{1}=0 \\
\left(-\frac{\partial^{2}}{\partial \rho^{2}}+f^{2}+f^{\prime}\right) R_{2}+\frac{1}{\rho}\left(-\frac{\partial}{\partial \rho}+f\right) R_{2}+\frac{j_{z}^{2}}{\rho^{2}} R_{2}=0 .
\end{gathered}
$$

The first order derivatives in these equations can be eliminated using the substitution

$$
R_{1}=\frac{1}{\sqrt{\rho}} F_{1}(\rho), \quad R_{2}=\frac{1}{\sqrt{\rho}} F_{2}(\rho) .
$$

$$
\begin{aligned}
& {\left[-\frac{\partial^{2}}{\partial \rho^{2}}+\left(f-\frac{1}{2 \rho}\right)^{2}-\left(f-\frac{1}{2 \rho}\right)^{\prime}+\frac{j_{z}^{2}}{\rho^{2}}\right] F_{1}=0,} \\
& {\left[-\frac{\partial^{2}}{\partial \rho^{2}}+\left(f+\frac{1}{2 \rho}\right)^{2}+\left(f+\frac{1}{2 \rho}\right)^{\prime}+\frac{j_{z}^{2}}{\rho^{2}}\right] F_{2}=0 .}
\end{aligned}
$$


$F_{1,2}(\infty)=0$ and equations $(28),(29)$ can be treated as equations on a half line. Let us rewrite these equations in the following form

$$
\begin{aligned}
& {\left[a^{+} a+\frac{j_{z}^{2}}{\rho^{2}}\right] F_{1}=0} \\
& {\left[b b^{+}+\frac{j_{z}^{2}}{\rho^{2}}\right] F_{2}=0}
\end{aligned}
$$

where

$$
a^{ \pm}=\mp \frac{\partial}{\partial \rho}+f-\frac{1}{2 \rho}, \quad b^{ \pm}=\mp \frac{\partial}{\partial \rho}+f+\frac{1}{2 \rho} .
$$

Note that we are interested in the square integrable so- lutions of equations (30), (31). But these equations have not square integrable solutions. Indeed, the eigenvalues of the operators $a^{+} a$ and $b b^{+}$which act in the space of the square integrable functions are positive including also the zero eigenvalue. Thus, the eigenvalues of the operators $a^{+} a+j_{z}^{2} / \rho^{2}$ and $b b^{+}+j_{z}^{2} / \rho^{2}$ are positive without the zero eigenvalue. Therefore, equations (30), (31) do not have the square integrable solutions. Thus, we can conclude that for the electron moving in the magnetic field of straight current the SUSY is broken.

\section{ACKNOWLEDGMENT}

V.M.T. thanks the Bohdan Shypyliavyi Foundation for the financial support.
[1] H. Nicolai, J. Phys. A 9, 1497 (1976).

[2] E. Witten, Nucl. Phys. B 185, 513 (1981).

[3] L. E. Gendenshteyn, N. V. Krive, Usp. Fiz. Nauk. 146, 553 (1985).

[4] F. Cooper, A. Khare, U. Sukhatme, Phys. Rep. 251, 267 (1995).

[5] G. Junker, Supersymmetric Methods in Quantum and Statistical Physics (Springer, Berlin, 1996).

[6] E. D'Hoker, L. Vinet, Phys. Lett. B 137, (1984).

[7] L. E. Gendenshteyn, Yad. Fiz. 41, 261 (1985).

[8] Yu. A. Sitenko, Yad. fiz. 51(5), 1416 (1990).

[9] V. M. Tkachuk, S. I. Vakarchuk, J. Phys. Stud. 1, 39 (1996).

[10] V. M. Tkachuk, S. I. Vakarchuk, Phys. Lett. A 228, 141 (1997).

[11] A. Nikitin, preprint JINR E2-96-369 (Dubna, 1996).

[12] A. Nikitin, in Group21, Physical applications and mathematical aspects of geometry, groups and algebras. Vol. 1, edited by H.-D. Doebner, V. Sherer, P. Natterman,
(World Scientific, Singapoore, 1997), p. 509.

[13] L. V. Hau, J. A. Golovchenko, M. M. Burns, Phys. Rev. Lett. 75, 1426 (1995).

[14] R. Blumel, K. Dietrich, Phys. Rev. A 43, 22 (1991).

[15] A. I. Voronin, Phys. Rev. A 43, 29 (1991).

[16] M. Müller, K. Dietrich, Phys. Rev. A 542577 (1996).

[17] F. Bentosela, R. M. Cavalcanti, P. Exner, V. A. Zagrebnov, preprint math-ph/9903030 (1999).

[18] F. Bentosela, P. Exner, V. A. Zagrebnov, preprint mathph/9810016 (1998).

[19] Y. Aharonov, A. Casher, Phys. Rev. A 192641 (1979).

[20] M. Loss, H.-T. Yau, Commun. Math. Phys. 104, 283 (1986).

[21] C. Adam, B. Muratori, C. Nash, preprint hepth/9903040, Phys. Rev. D (to appear).

[22] C. Adam, B. Muratori, C. Nash, preprint hepth/9910139.

[23] J. Fröhlich, E. Lieb, M. Loss, Commun. Math. Phys. 104, 251 (1986).

\title{
ПОРУШЕНА СУПЕРСИМЕТРІЯ ЕЛЕКТРОНА В МАГНЕТНОМУ ПОЛІ ПРЯМОГО СТРУМУ
}

\author{
В. М. Ткачук, С. I. Вакарчук \\ Львівсъкий національний університет ім. І. Франка, \\ кафедра теоретичнӧ̈ фізики, \\ вул. Драгоманова, 12, Львіб, 79005, Украӥна \\ E-mail: thachuk@ktf.franko.lviv.ua
}

Гамільтоніян Паулі для електрона, що рухається в магнетному полі прямого струму, володіє $N=3$ суперсиметрією. Ми вивчаємо рівняння для основного стану з нульовою енергією електрона, що рухається в цьому полі. Показано, що це рівняння не має квадратично інтегровних розв'язків і, таким чином, суперсиметрія є порушеною. 